\title{
Tumor Resection May Improve Survival in Patients With Soft Tissue Sarcoma Aged 75 Years and Older
}

\author{
TOMOKI NAKAMURA, TORU OI, KUNIHIRO ASANUMA, TOMOHITO HAGI and AKIHIRO SUDO \\ Department of Orthopaedic Surgery, Mie Graduate School of Medicine, Mie, Japan
}

\begin{abstract}
Background/Aim: This study aimed to analyze the decision-making process of treatment and the clinical outcomes of patients with STS aged 75 years and older. Patients and Methods: Overall, 72 patients were reviewed. The mean and median ages were 82 and 81 years, respectively. Results: Of the 72 patients, 17 did not undergo surgical resection. Age was related to the decision to pursue surgical treatment. Resection was performed in 55 patients. The 5-year overall survival was 51.8\%. Patients with an American Society of Anesthesiologists physical status (ASA-PS) score of 3 had a worse survival. Twenty-five patients developed metastasis. The median survival time after metastasis in 12 patients who received metastasectomy and/or lung radiofrequency ablation was superior (18.2 months) to that of the remaining 13 patients without any treatment (3.2 months). Conclusion: Surgical treatment should be considered in elderly patients if their physical condition is not poor. ASAPS score may be a tool to indicate physical condition.
\end{abstract}

The proportion of the elderly population is rapidly increasing in Japan. Twenty-seven percent and $13.8 \%$ of Japanese citizens were aged $>65$ years and $>75$ years in 2017, respectively (reported by cabinet office, Government of Japan). In recent years, the incidence of soft tissue sarcoma (STS) in elderly patients has been increasing (1). Surgical resection with or without adjuvant radiotherapy is the standard treatment for STS (2-4). However, less aggressive treatment is often recommended for elderly patients because of their comorbidities and their decision to refuse surgery. Recently, several authors have suggested that older age itself was not associated with decreased disease-specific survival

This article is freely accessible online.

Correspondence to: Tomoki Nakamura, Department of Orthopaedic Surgery, Mie University Graduate School of Medicine, 2-174 Edobashi, Tsu-city, Mie, 514-8507, Japan. Tel: +81 592315022, Fax: +81 592315211, e-mail: tomoki66@clin.medic.mie-u.ac.jp

Key Words: Soft tissue sarcoma, elderly, survival, American Society of Anesthesiologists physical status, metastasectomy. or prognosis in elderly patients with STS who underwent surgical treatment (3-5). Furthermore, when patients develop metastasis, the treatment should be limited, because chemotherapy has not been shown to increase survival after resection of lung metastases and carries high risk of adverse events in elderly patients (6).

This study aimed to analyze the decision-making process of treatment and the clinical outcome of patients with STS aged 75 years and older. We emphasize that surgical treatment including metastasectomy should be considered if the physical condition of the patient is not poor.

\section{Materials and Methods}

A total of 72 patients aged 75 years or older who presented to our hospital between April 2007 and March 2017 were reviewed. Patients presenting with local recurrence at diagnosis were excluded. The pretreatment work-up included lung, abdomen, and pelvis computed tomography (CT) scans. The histopathological diagnoses and tumor grades were determined using the French Federation of Cancer Centers Sarcoma Group (FNCLCC) system for all patients and were reviewed by independent pathologists. Surgical margins were microscopically evaluated in all patients. The study protocol was approved by the Institutional Review Board. The need for informed consent was waived because of the retrospective nature of this study.

Statistical analysis. Statistical associations of the clinicopathological factors were evaluated using the Mann-Whitney $U$-test for quantitative data and the $\chi^{2}$ or Fisher's exact test for qualitative data. The duration of overall survival was defined as the interval between the date of initial treatment for the primary tumor and that of allcause mortality. The duration of disease-specific survival was defined as the interval between the date of initial treatment for the primary tumor and that of sarcoma-related death. The survival curves were constructed using the Kaplan-Meier method. The log-rank test and Cox hazard model were used to compare the survival times. $p$ Values $<0.05$ were considered significant in all statistical analyses.

\section{Results}

Patients. The mean and median ages of the 72 patients reviewed in this study were 82 and 81 years, respectively (range $=75-94$ years). Four patients were referred to our hospital for the management of inappropriate surgery at another hospital. 
Of the 72 patients, 17 did not undergo surgical resection (Figure 1). Age was related to the decision to pursue surgical treatment $(p<0.01)$ (Table I). Of 17 patients, six refused surgery, five were deemed irresectable by the multidisciplinary team, three had a high risk of death due to comorbidities, two had metastatic disease, and one patient died of stomach cancer during the assessment period. Also, out of these 17 patients, 11 received palliative radiotherapy.

Resection was performed in 55 patients. Four patients underwent additional tumor resection after inappropriate resection at another hospital. The mean and median ages were 81 years and 80 years, respectively (range $=75-94$ years). The cohort included 28 male and 27 female patients. The mean and median tumor sizes were 9.4 and $8 \mathrm{~cm}$, respectively (range $=2-27 \mathrm{~cm}$ ). The mean and median follow-up periods after surgery were 36 months and 25 months, respectively. Primary tumor sites included the thigh $(n=20)$, lower leg $(n=9)$, back $(n=5)$, upper arm $(n=5)$, buttock $(n=3)$, forearm $(n=3)$, and other regions $(n=10)$. The tumors were histologically-classified as myxofibrosarcomas $(n=14)$, undifferentiated pleomorphic sarcomas (UPSs) $(n=12)$, well-differentiated liposarcomas $(n=8)$, leiomyosarcomas $(n=9)$, malignant peripheral nerve sheath tumors $(n=4)$, de-differentiated liposarcomas $(n=4)$, and others $(n=4)$. Ten patients were classified as grade 1 (low grade) according to the FNCLCC grading system. The remaining 45 patients were classified as grade 2 or 3 (high grade). Two patients had distant metastases at initial presentation.

Comorbidities. Thirty-six patients received medication for hypertension. Ten received anti-coagulant medication because of ischemic disease, previous myocardial infarction or cerebrovascular accident. Nine patients had a history of cancer (past=6; present=3). Nine patients received medication for diabetes mellitus. According to the American Society of Anesthesiologists physical status (ASA-PS) scoring system, the score was 3 in seven patients, 2 in 41 patients, and 1 in seven patients.

Surgical treatment. Wide surgical margins were achieved in 37 patients, including two patients with amputation. Marginal margin was achieved in 11 patients and intralesional in five patients. The remaining two patients received autologous irradiated recycled bone graft because of the suspicion of bone invasion. Five patients received adjuvant radiotherapy. There were no patients who received neo- and/or adjuvant chemotherapy.

Of 55 patients, 10 had complications after surgery. Wound dehiscence was observed in four patients, infection in two, delirium in two, and skin necrosis in two. Additional surgical treatment due to complications was required in six patients. No patients died due to complications.
Outcome. At the final follow-up, 29 patients were alive with no disease, four were alive with disease, 17 had died of sarcoma, five had died of other diseases. Of five patients who died of other diseases, the cause of death was heart failure in two patients, and rupture of aorta, pancreatic cancer and pneumonia in one patient each. No patients died within 30 days of surgery.

The 3-year and 5-year overall survival rates in the 55 patients were $58.6 \%$ (95\% confidential interval $(\mathrm{CI})=43.1-74)$ and $51.8 \%$ (95\% $\mathrm{CI}=35.6-68.1)$, respectively. When we divided the patients into three groups according to ASA-PS score, patients with a score of 3 had worse survival, compared to those with a score of $2(p=0.02)$. Age, tumor size, gender, and tumor grade were not related to overall survival (Table II).

The 3-year and 5-year disease-specific survival rates in the 55 patients were $64.4 \%(95 \% \mathrm{CI}=49.1-79.8)$ and $60.4 \%$ (95\% CI=44.1-76.7), respectively.

Local recurrence occurred in nine patients. Of these, six underwent surgical resection, one patient received radiotherapy, one patient refused surgery and received palliative therapy and the remaining patient received palliative therapy due to synchronous multiple lung metastases. Twentyfive patients developed metastasis. Metastasectomy and/or radiofrequency ablation were performed in nine patients. Metastasectomy or RFA and chemotherapy were performed in three patients. The remaining 13 patients, including three who refused surgical treatment received palliative therapy. The median survival time after metastasis in 12 patients who received metastasectomy and/or RF ablation was 18.2 months, while median time in 13 patients without metastasectomy and/or RF ablation was 3.2 months $(p<0.001)$ (Figure 2).

\section{Discussion}

In our cohort, 55 (76\%) of 72 patients aged 75 or older with STS underwent surgical tumor resection. Age was related to the decision to pursue surgical treatment. Patients with ASA scores of 3 had a worse survival than those with scores of 2 . There were no patients who died due to complications. Twentyfive $(45 \%)$ of 55 patients developed metastasis. In 25 patients with metastasis, 12 underwent metastasectomy and/or RFA.

The proportion of patients who received no treatment is higher among elderly patients. Hoven-Gondrie et al. reported that among patients with extremity STS who were aged $>80$ years $(n=371), 14.8 \% \quad(n=55)$ received no treatment compared to $2.7 \%$ of patients aged $<65$ years $(7)$. In the present study, 11 of 17 patients did not undergo surgical resection because of the decision of the physicians. The remaining six patients refused surgery due to the their own and/or their family's decision in spite of having resectable STS. The decision to refrain from sarcoma treatment was primarily disease-related and was less often based on poor general health status $(7,8)$. In addition to those causes, it 


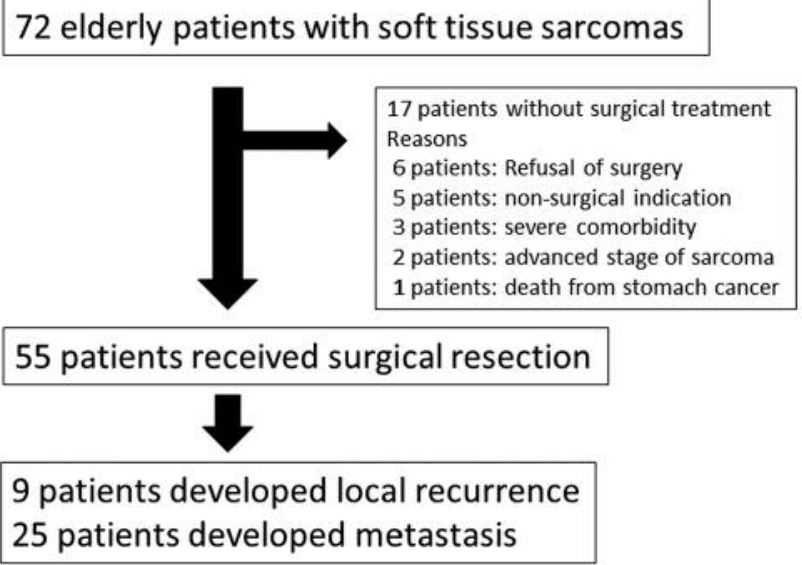

Figure 1. Flow chart of the treatment.

Table I. The affection of decision of surgical treatment.

\begin{tabular}{lccc}
\hline Variables & \multicolumn{2}{c}{ Surgery } & $p$-Value \\
\cline { 2 - 3 } & Yes & No & \\
\hline Age & & & \\
$\quad$ Mean (years) & 80.5 & 85.7 & 0.0004 \\
Gender & & & \\
$\quad$ Male & 28 & 8 & $>0.99$ \\
Female & 27 & 9 & \\
Size & & & 0.42 \\
$\quad$ Mean (cm) & 9.4 & 9.6 & 0.75 \\
ASA-PS & & & \\
Mean & 2 & 1.9 & 0.4 \\
$\quad$ score of 3 & 7 & 4 & \\
Grade & & & \\
Low & 10 & 1 & \\
High & 45 & 16 & \\
\hline
\end{tabular}

ASA-PS: American Society of Anesthesiologists physical status.

may become more complicated to optimize sarcoma care because of patient and family decisions.

Although the ASA-PS score was originally designed as a uniform system for anesthesiologists to convey information about the patient's overall health status, it has been shown to accurately predict perioperative morbidity and mortality as well. Furthermore, numerous studies have suggested that the ASA-PS score may provide prognostic information in many cancer surgeries including bone and soft tissue sarcoma (9-11). In the present study, ASA-PS score was a prognostic factor for overall survival. Although elderly patients with STS have a poor survival, this trend may have been caused by undertreatment in this population $(4,12)$. Okamoto et al.

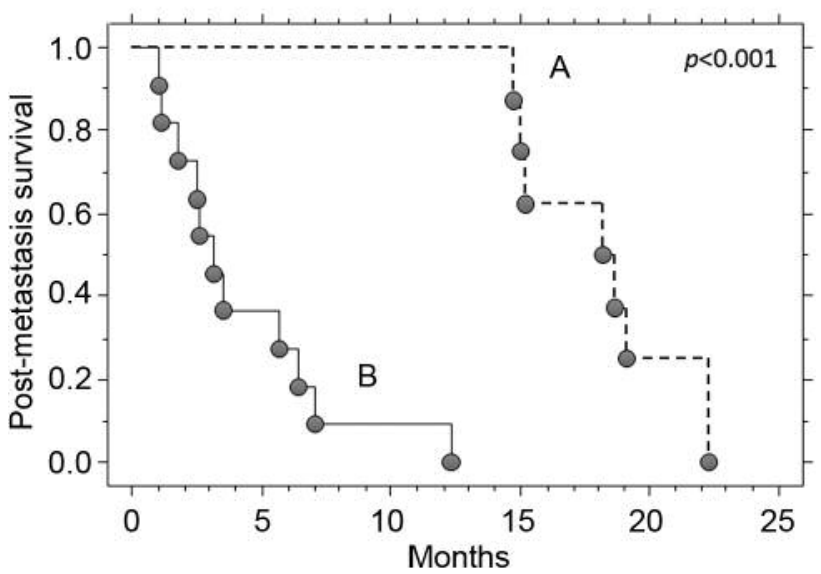

Figure 2. Kaplan-Meier curve for post-metastasis survival. A: Patients with metastasectomy and/or radiofrequency ablation. B: Patients without metastasectomy and/or radiofrequency ablation.

Table II. The prognostic factor for overall survival.

\begin{tabular}{llll}
\hline Variables & HR & $95 \%$ CI & $p$-Value \\
\hline $\begin{array}{l}\text { Age } \\
\text { Years }\end{array}$ & 1.073 & $0.984-1.17$ & 0.11 \\
$\begin{array}{l}\text { Gender } \\
\text { Male }\end{array}$ & 1 & & \\
$\quad$ Female & 0.749 & $0.327-1.717$ & 0.49 \\
Size & 1.006 & $0.932-1.087$ & 0.87 \\
cm & 1 & & \\
Grade & 4.549 & $0.609-34.011$ & 0.14 \\
Low & 1 & & \\
High & 0.274 & $0.095-0.789$ & 0.02 \\
ASA-PS & 0.139 & $0.053-1.43$ & 0.12 \\
3 & & & \\
2 & & & \\
1 & & & \\
\hline
\end{tabular}

ASA-PS: American Society of Anesthesiologists physical status; HR: hazard risk; 95\%CI: 95\% confidence interval.

reported that patients with STS aged 80 and older that had surgical treatment had better survival rates than those who did not undergo surgery. They reported that one- and two-year sarcoma-specific survival rates were $25 \%$ and $0 \%$, respectively in the conservative therapy group and $90.9 \%$ and $75.4 \%$, respectively in the surgical treatment group (4). We believe that surgical treatment should be considered in elderly patients if their physical condition is not poor, as in ASA-PS 3 or 4.

Of patients with extremity sarcoma, approximately $30 \%$ will have metastatic disease at some point in the course of their disease (13). In the present study, 25 (45\%) of 55 patients developed metastasis. Chemotherapy has not been shown to increase survival after resection of lung metastases 
$(14,15)$. Surgical resection of metastases has been thought to be the only therapeutic option to enhance survival (15-17). However, metastasectomy in elderly sarcoma patients has been performed with a great deal of hesitation because of the high incidence of surgical mortality and morbidity. Recently, lung radiofrequency (RF) ablation has been accepted as a relatively safe and useful therapeutic alternative for the treatment of unresectable non-small cell lung cancer and lung metastases $(6,18)$. We applied RF ablation in 4 patients with lung metastasis.

This study had some limitations. Its retrospective nature was a major limitation. The small number of patients was also another limitation. Future large-scale studies are required to validate these results.

In conclusion, age was related to the decision to pursue surgical treatment. Patients with ASA-PS scores of 3 had a worse survival, compared with those with scores of 2 . There were no patients who died due to complications. Metastasectomy, including RF ablation may improve survival in patients with metastases. We believe that surgical treatment should be considered in elderly patients if their physical condition is not poor. ASA-PS score may be a tool to indicate physical condition.

\section{Conflicts of Interest}

The Authors declare that there were no conflicts of interest and no financial support regarding the present study.

\section{References}

1 Japanese Orthopaedic Association Musculoskeletal Tumor Committee. Soft tissue tumor registry in Japan 2012. Tokyo: National Cancer Center; 2012.

2 Clark MA, Fisher C, Judson I and Thomas JM: Soft-tissue sarcoma in adults. N Engl J Med 353: 701-711, 2005.

3 Yoneda Y, Kunisada T, Naka N, Nishida Y, Kawai A, Morii T, Takeda K, Hasei J, Yamakawa Y and Ozaki T: Favorable outcome after complete resection in elderly soft tissue sarcoma patients: Japanese Musculoskeletal Oncology Group study. Eur J Surg Oncol 40: 49-54, 2014.

4 Okamoto M, Yoshimura Y, Aoki K, Kito M, Tanaka A, Suzuki $\mathrm{S}$, Takazawa A, Isobe $\mathrm{K}$ and Kato $\mathrm{H}$ : Clinical outcome of patients 80 years of age and older with soft tissue sarcoma. J Orthop Sci 22: 951-957, 2014.

5 Tsuchie H, Emori M, Nagasawa H, Miyakoshi N, Murahashi Y, Mizushima E, Yamashita T and Shimada Y: The prognostic significance of surgical treatment for excessive elderly patients with soft tissue sarcoma. Int J Clin Oncol 23: 775-782, 2018.

6 Nakamura T, Matsumine A, Yamakado K, Takao M, Uchida A and Sudo A: Clinical significance of radiofrequency ablation in elderly patients with lung metastases from musculoskeletal sarcomas. J Can Res Ther 9: 219-223, 2013.
7 Hoven-Gondrie ML, Bastiaannet E, Ho VKY, van Leeuwen BL, Liefers GJ, Hoekstra HJ and Suurmeijer AJ: Worse survival in elderly patients with extremity soft-tissue sarcoma. Ann Surg Oncol 23: 2577-2585, 2016.

8 Farshadpour F, Schaapveld M, Suurmeijer AJ, Wymenga AN, Otter R and Hoekstra HJ: Soft tissue sarcoma: why not treated? Crit Rev Oncol Hematol 54: 77-83, 2005.

9 Cullen DJ, Apolone G, Greenfield S, Guardagnoli E and Cleary P: ASA Physical Status and age predict morbidity after three surgical procedures. Ann Surg 220: 3-9, 1994.

10 Kang HW, Seo SP, Kim WT, Kim YJ, Yun SJ, Lee SC, Choi YD, Ha YS, Kim TH, Kwon TG, Byun SS, Jeh SU and Kim WJ: Impact of the ASA Physical Status score adjuvant chemothetapy eligibility and survival of upper tract urothelial carcinoma patients: a multicenter study. J Korean Med Sci 32: 352-342, 2017.

11 Iwai T, Hoshi M, TakadaJ, Oebisu N, Aono M, Takami M, Ieguchi $\mathrm{M}$ and Nakamura $\mathrm{H}$ : Prognostic factors for elderly patients with primary malignant bone and soft tissue tumors. Oncol Lett 10: 1799-1804, 2015.

12 Al-Refaie WB, Habermann EB, Dudeja V, Vickers SM, Tuttle TM, Jensen EH and Virnig EA: Extremity soft tissue sarcoma care in the elderly: insight into the generalizability of NCI Cancer Trials. Ann Surg Oncol 17: 1732-1738, 2010.

13 Antunes M, Bernardo J, Salete M, Prieto D, Eugénio L and Tavares P: Excision of pulmonary metastases of osteogenic sarcoma of the limbs. Eur J Cardiovasc Surg 15: 592-596, 1999.

14 Lanza LA, Putman JB Jr, Benjamin RS and Roth JA: Response to chemotherapy does not predict survival after resection of sarcomatous pulmonary metastases. Ann Thorac Surg 51: 219224, 1991.

15 Casper ES, Gaynor JJ, Harrison LB, Panicek DM, Hajdu SI and Brennan MF: Preoperative and postoperative adjuvant combination chemotherapy for adults with high-grade soft tissue sarcoma. Cancer 127: 1407-141, 1994.

16 van Geel AN, Pastorino U, Jauch KW, Judson IR, van Coevorden F, Buesa JM, Nielsen OS, Boudinet A, Tursz T and Schmitz PI: Surgical treatment of lung metastases: The European organization for research and treatment of cancer-soft tissue and bone sarcoma group study of 255 patients. Cancer 77: 675-682, 1996.

17 Smith R, Pak Y, Kraybill W and Kane III JM: Factors associated with actual long-term survival following soft tissue sarcoma pulmonary metastasectomy. Eur J Surg Oncol 35: 356-361, 2009.

18 Nakamura T, Matsumine A, Yamakado K, Matsubara T, Takaki H, Nakatsuka A, Takeda K, Ado D, Shimizu T and Uchida A: Lung radiofrequency ablation in patients with pulmonary metastases from musculoskeletal sarcomas. Cancer 115: 3774-3781, 2009.

Received November 20, 2018

Revised November 26, 2018

Accepted November 28, 2018 